\title{
Utilidad de los certificados de defuncion en colombia en la evaluacion de las causas de muerte por cancer de endometrio
}

\author{
Usefulness of death certificates in colombia \\ when evaluating the causes of death from endometrial cancer
}

\author{
Mario Arturo González Mariño*
}

\begin{abstract}
Resumen
Objetivo: Revisar las causas directas de muerte por cáncer de endometrio en Colombia según datos de los certificados de defunción en el año 2008. Material y Métodos: Se revisaron las causas directas de muerte en pacientes cuyo código de causa básica de defunción fue el de tumor maligno del endometrio(C54.1) según el registro de defunciones del Departamento Nacional de Estadística de Colombia (DANE) en el año 2008. Se evalúa su distribución por causa directa de muerte, edad, nivel educativo, estado civil, seguridad social y sitio de defunción. Resultados: Se registraron 157 fallecimientos. Las principales causas directas de muerte fueron Insuficiencia respiratoria en $38(24,2 \%)$, adenocarcinoma de endometrio en $29(18,4 \%)$, falla orgánica múltiple en $26(16,5 \%)$ y paro cardiorespiratorio en $16(10,1 \%)$. Conclusiones: Se evidencian fallas en el correcto diligenciamiento de los registros de defunción en Colombia. Se debe tener en cuenta la Clasificación internacional de enfermedades (CIE-10) para mejorar su calidad y aprovechar la información de este documento.
\end{abstract}

Palabras clave: causas de muerte, neoplasias uterinas, registros de mortalidad, neoplasias endometriales (fuente: DeCS, BIREME)

\begin{abstract}
Objective: Examine the direct causes of death from endometrial cancer according to data from death certificates in 2008. Equipment and Method: The study examined the direct cause of death in patients whose official cause of death was a malignant endometrial tumour (C54.1) according to death registration data from the Departamento Nacional de Estadística de Colombia (The National Department of Statistics in Colombia) in 2008. The study assessed the distribution by direct cause of death, age, level of education, marital status, social security and place of death. Results: 157 deaths were registered. The main direct causes of death were respiratory failure in 38 cases $(24.2 \%)$, endometrial adenocarcinoma in 29 cases (18.4\%), multiple organ failure in 26 cases $(16.5 \%)$ and 16 cases of cardiac, respiratory arrest (10.1\%). Conclusions: Inaccuracies were found in the death registers in Colombia where information was not filled out correctly. The International Classification of Diseases (ICD), as well the information in this study, must be taken in account in order to improve the accuracy of the death registers.
\end{abstract}

Keywords: causes of death, uterine neoplasms, death certificates, endometrial neoplasms

* MD, Ginecólogo Oncólogo y Mastólogo, Profesor Asociado Universidad Nacional de Colombia, Docente Fundación Universitaria San Martín, Especialista Hospital Central de la Policía, Bogotá, Colombia.

Correo electrónico: marioar90@hotmail.com

Recibido: 13/01/14-Revisado: 29/09/2014-Aceptado: 30/03/2015 


\section{Introducción}

El cáncer de endometrio es el cáncer más frecuente en los Estados Unidos de América ${ }^{[1]}$. En Colombia, ocupa el segundo lugar en frecuencia entre las enfermedades malignas ginecológicas, después del carcinoma de cuello uterino con una tasa de incidencia estandarizada por edad(según población mundial) de 5,9x100000 mujeres por año y una tasa de mortalidad media anual estandarizada por edad(según población mundial) de $1,7 \times 100000$ mujeres por año ${ }^{[2]}$.

El tipo histológico más frecuente es el tipo endometriode. La mayoría de los casos (75\%) se diagnostican en estadio I cuyo pronóstico es bastante bueno con una supervivencia de $90 \%$ a 5 años. La mayoría de estos canceres ocurren después de la menopausia, pero un $25 \%$ se presentan en etapa pre-menopáusica ${ }^{[3]}$.

Las causas directas de esta mortalidad no han sido plenamente identificadas. Al revisar las bases de datos Pubmed y Lilacs no se encuentra ningún artículo sobre el tema en Colombia. Es importante aprovechar los registros de defunción en el análisis de mortalidad por cáncer endometrial. Para definir las causas de muerte se buscaron y evaluaron las causas directas anotadas en el registro de defunciones del DANE para tumor maligno del endometrio en el año $2008^{[4]}$.

\section{Materiales y métodos}

Se revisaron las causas directas de muerte en todos los fallecimientos con código de tumor maligno del endometrio (C54.1) en el registro de defunciones del DANE en el año 2008. Se evalúa su distribución por causa directa de muerte, edad, nivel educativo, estado civil, seguridad social y sitio de defunción.

\section{Resultados}

En Colombia se registraron 157 fallecimientos con causa básica de tumor maligno del endometrio (C54.1) durante el año 2008 .La mayoría de los casos se presentaron en Bogotá D.C, 58 casos $(36,9 \%)$ y por departamentos, Antioquia con 23 casos $(14,6 \%)$ y Valle del Cauca con $15(9,5 \%)$.

El promedio de edad en que ocurrieron las muertes fue 67,7 años (31-97años). En menores de 50 años los fallecimientos fueron $7(4,4 \%)$ y 38 entre 50 y 60 años $(24,2 \%)$, En el análisis de estadística descriptiva se encontraron dos modas, 62 y 77 años (7 casos cada uno). Las principales causas directas de muerte fueron Insuficiencia respiratoria o ventilatoria en $38(23,7 \%)$, adenocarcinoma de endometrio en $29(18,1 \%)$, falla orgánica múltiple o multi-sistémica en $26(16,2 \%)$ y paro cardiorespiratorio en $16(10 \%)$.

En la Tabla 1, se detallan los resultados de las defunciones según sitio de fallecimiento, estado civil, nivel educativo y seguridad social.

\section{Discusión}

Las causas de muerte extraídas del certificado de defunción, sufren de un alto grado de imprecisión para atribuir la causa
Tabla 1. Principales resultados de variables analizadas en los registros de defunción.

\begin{tabular}{lcc}
\hline Característica & $\mathrm{N}=157$ & $\%$ \\
\hline $\begin{array}{l}\text { Sitio de fallecimiento } \\
\text {-Hospital o clínica }\end{array}$ & 85 & 54,1 \\
-Casa o domicilio & 72 & 45,8 \\
\hline Estado civil & & \\
-Casadas & 49 & 31,2 \\
-Solteras & 38 & 24,2 \\
-Separadas o divorciadas & 13 & 8,2 \\
-Viudas & 38 & 24,2 \\
-Otro & 8 & 5,0 \\
-Sin información & 11 & 7,0 \\
\hline
\end{tabular}

\section{Nivel de educación}

-Básica primaria

$72 \quad 45,8$

-Básica secundaria

15

9,5

- Media académica o clásica

14

-Profesional

5

8,9

-Ninguna

3,1

-Sin información

8,9

-otros

14

21,0

\section{Seguridad social}

-Régimen contributivo

33

2,5

-Régimen subsidiado

-Otros

-No asegurados

-Sin información

4

2,5

directa de muerte ${ }^{[5]}$. Así, se siguen empleando causas como paro cardiorespiratorio, el cual poco dice de la causa real de muerte, se registran condiciones que no se encuentran en la Clasificación Internacional de enfermedades (CIE 10) ${ }^{[6]}$ como falla orgánica multisistémica, falla multiorgánica o síndrome de falla multisistémica ${ }^{[4]}$ y se coloca la causa básica de muerte como la causa directa ignorando las indicaciones expuestas en el formulario de que se deben registrar en un renglón diferente. De esta manera, la causa básica, registrada en el último renglón de la parte I del certificado de defunción, es la que origina algunas complicaciones (consecuencias de la causa básica) que deben ser registradas en las líneas de arriba(a, b, y c). La última de las complicaciones o consecuencias, registrada en la línea a), es llamada causa terminal o inmediata o causa directa de muerte ${ }^{[7,8]}$. A pesar de esto, para el diagnóstico de cáncer lo que se ha visto es que los registros son más confiables que los de otras patologías (9)

Dado que el certificado de defunción tiene propósitos estadísticos ${ }^{[7]}$ se hace necesario establecer normas para el 
R.F.S Revista Facultad de Salud

Enero-Junio de 2015;7(1): 66-68
Utilidad de los certificados de defunción en colombia en la evaluacion de las causas de muerte por cancer de endometrio / Mario Arturo González Mariño diagnóstico de las causas directas de muerte que hagan más aprovechable este importante instrumento. La intención del Ministerio de salud de extender el formulario sistematizado en el marco del Sistema Integral de Información de la Protección Social - SISPRO, que desarrolló el Registro Único de Afiliados - RUAF, del cual hace parte el módulo de nacimientos y defunciones ${ }^{[10]}$ podría utilizarse en mejorar la calidad en el registro de la causa directa de muerte.

En las otras variables evaluadas, el porcentaje de afiliadas al régimen contributivo o subsidiado $(86,6 \%)$ fue superior al total nacional presente en el año $2008(83,2 \%)^{[11]}$.

A pesar de que la mayor frecuencia en el estado civil correspondió a las casadas, se evidencia un alto número de viudas, lo cual se puede relacionar con la mayor expectativa de vida de las mujeres en Colombia (70,67años en hombres y 77,51 en mujeres) ${ }^{[12]}$.

La educación básica primaria fue el nivel educativo más frecuente en estas mujeres con el 45,8\%. Aunque el nivel educativo y la afiliación al régimen de seguridad social pueden tener asociación con el nivel socio económico, este factor no se ha consolidado como factor de riesgo en cáncer de endometrio ${ }^{[13]}$. A pesar de que inicialmente se reportó un mayor riesgo de desarrollar esta neoplasia en mujeres con nivel socio económico alto ${ }^{[14]}$, esto no se ha podido replicar en investigaciones subsecuentes ${ }^{[15,16]}$.

La defunción ocurrió con alta frecuencia en la casa o domicilio. Al respecto, como se preguntan Ochoa y Montoya sería interesante saber si estas eran personas en estado terminal o se trató de personas sin acceso a los servicios de salud ${ }^{[17]}$.

\section{Conclusiones}

Las principales denominaciones anotadas en los registros de defunción como causa directa de muerte por cáncer endometrio fueron insuficiencia respiratoria, adenocarcinoma de endometrio, falla orgánica múltiple y paro cardiorespiratorio. Se evidencian fallas en el correcto diligenciamiento de los registros de defunción en Colombia. Se debe tener en cuenta la Clasificación internacional de enfermedades (CIE-10) para mejorar su calidad y aprovechar la información de este documento.

\section{Referencias}

1. Jemal A, Siegel R, Xu J, Ward E. Cancer statistics, 2010.CA Cancer J Clin 2010;60:277-300.

2. Registro poblacional de cáncer de Cali, facultad de salud Universidad del Valle [Internet]. Disponible en: rpcc.univalle.edu.co .Consultado en Julio 23, 2012.

3. Plataniotis G, Castiglione M; ESMO Guidelines Working Group. Endometrial cancer: ESMO Clinical Practice Guidelines for diagnosis, treatment and follow-up. Annals of Oncology 2010; 21 (Supplement 5): v4 1-v45.
4. Departamento Nacional de Estadística (DANE). Registro de defunciones año 2008, Bogotá. Colombia.

5. Vecino A.I. Precisión en el diligenciamiento de los certificados de defunción en el Instituto Nacional de Cancerología, Colombia. Rev Colomb Cancerol 2006; 10(3): 170-182.

6. International Classification of Diseases (ICD) [Internet]. Disponible en http://www.who.int/classifications/icd/en/ - Consultado Julio 22 de 2012.

7. Agudelo B, Henao JA, Méndez OA. El certificado de defunción: Un instrumento para la vigilancia en salud pública [Internet]. Disponible en http://www.nacer. udea.edu.co/pdf/libros/librol/elcertificadode defuncion.pdf, Consultado Julio 14 de 2012.

8. Secretaría Departamental de Salud. El ABC de las estadísticas vitales, Año 1 - Número 001 - Mayo 2009 [Internet]. Disponible en www.valledelcauca.gov.co/salud/descargar.php?id=1219 Consultado Julio 14 de 2012.

9. Kircher T, Nelson J, Burdo H. The autopsy as a measure of accuracy of the death certificate. N Engl J Med 1985; 313:1263-1269.

10. RUAF-Ministerio de Salud y la Protección Social. Bogotá, Colombia [Internet]. Disponible en: http:// www.minsalud.gov.co/salud/Paginas/Aplicaci\% C3\%B3nNacimientosyDefunciones-RUAF.aspx . Consultado Junio 14 de 2012.

11. Ministerio de salud y Protección Social. Cobertura en Salud. Bogotá, Colombia. [Internet]. Disponible en: http://www.minsalud.gov.co/salud/Paginas/ CoberturasdelR\%C3\%A9gimenSubsidiado.aspx . Consultado Junio 16 de 2012.

12. Departamento Nacional de Estadística (DANE). Bogotá, Colombia [Internet]. Disponible en: http://www.dane. gov.co/files/BoletinProyecciones.pdf. Consultado Junio 16 de 2012.

13. Purdie DM, Green AC. Epidemiology of endometrial cancer. Best Practice and Research Clinical Obstetrics and Gynaecology 2001; 15(3):341-354.

14. Kelsey JL, LiVolsi VA, Holford TR et al. A case-control study of cancer of the endometrium. American Journal of Epidemiology 1982; 116: 333-342.

15. Shapiro S, Kaufman DW, Slone D et al. Recent and past use of conjugated estrogens in relation to adenocarcinoma of the endometrium. New England Journal of Medicine 1980; 303: 485-489.

16. Ewertz M, Schou G \& Boice JD Jr. The joint effect of risk factors on endometrial cancer. European Journal of Cancer and Clinical Oncology 1988; 24: 189-194.

17. Ochoa FL, Montoya LP. Mortalidad por cáncer en Colombia 2001. Revista CES Medicina 2004; 18(2):19-36. 